\title{
DERECHO Y POLÍTICAS AMBIENTALES EN CASTILLA Y LEÓN (SEGUNDO SEMESTRE 2016)
}

\author{
IÑIgO S ANZ RUBiALES \\ Catedrático de Derecho Administrativo \\ Universidad de Valladolid
}

\begin{abstract}
Sumario: 1. Introducción. 2. Protección de animales: el Decreto-Ley del Toro de la Vega. 3. Conservación y gestión del lobo. 3.1. El plan del lobo: correcciones y mejoras. 3.2. El necesario complemento: la regulación de las ayudas compensatorias por ataques de lobos. 4. Regulación de ayudas a la plantación de especies forestales de alto valor. 5. Planes y estrategias no vinculantes. 5.1. La II Estrategia de Educación Ambiental 2016-2020. 5.2. La Estrategia de Producción Ecológica 2016-2020. 5.3. La Estrategia de Regeneración Urbana.
\end{abstract}

\section{Introducción}

La actividad normativa ambiental de la Comunidad Autónoma en este segundo semestre de 2016 ha sido escasa. Destaca, sin dudas, el polémico Plan de Gestión del Lobo, especie que en el norte de Castilla goza de buena salud y que está en un proceso de expansión, hacia el sur y hacia otras Comunidades Autónomas. No obstante esta escasez de normas, sí que merece la pena destacar varios acuerdos de la Junta de Castilla y León, no normativos, no vinculantes, pero de una indudable trascendencia ambiental para la región, porque marcan las estrategias de educación ambiental, de producción ecológica y de regeneración urbana para los próximos años.

\section{Protección de animales: el Decreto-Ley del Toro de la Vega}

El Decreto-ley 2/2016, de 19 de mayo, por el que se prohíbe la muerte de las reses de lidia en presencia del público en los espectáculos taurinos populares y tradicionales en Castilla y León, convalidado por Resolución de 8 de junio de 2016, de la Presidencia de las Cortes de Castilla y León (BOCYL de 21 de junio de 2016), se dirigió a evitar la muerte por lanza del famoso "Toro de la Vega" de Tordesillas.

Como hemos señalado en otros comentarios legislativos anteriores, este tipo de medidas no se enmarca en modo alguno en la materia "medio ambiente", sino que responde 
propiamente a las competencias en materia de "ganadería" (condiciones de vida o muerte del ganado, de lidia en este caso) y de espectáculos públicos. Ahora bien, como consecuencia de la importante polémica que ha levantado la tradicional fiesta del Toro de la Vega, enfrentando a animalistas foráneos y vecinos partidarios de la fiesta, no se puede dejar de aludir a esta norma.

Sin perjuicio del contenido, llama la atención el instrumento utilizado por la Comunidad Autónoma para convertir dicho espectáculo en un encierro más: no deja de resultar paradójica la utilización del Decreto-Ley (que sólo puede usarse en caso de urgente necesidad) cuando el Toro de la Vega se ha venido celebrando anualmente, durante cerca de cinco siglos.

\section{Conservación y gestión del lobo}

\subsection{El plan del lobo: correcciones y mejoras}

El Decreto 14/2016, de 19 de mayo, (BOE del 23) aprueba el Plan de Conservación y Gestión del Lobo en Castilla y León. Este Plan viene a sustituir al anterior Plan de Conservación y Gestión del Lobo en Castilla y León aprobado por la Junta de Castilla y León por Decreto 28/2008, de 3 de abril. Como reconoce la Exposición de Motivos del Plan vigente, el derogado fue objeto de dos Sentencias de la Sala de lo ContenciosoAdministrativo del Tribunal Supremo, ambas de 22 de marzo de 2013, relativas a los recursos de casación planteados por la Comunidad de Castilla y León contra sendas sentencias de la Sala de los Contencioso-Administrativo del Tribunal Superior de Justicia de Castilla y León, que declararon nulos los artículos 4 apartado c), 8, 12. $1^{\circ}$ b) y $2^{\circ}, 14$ y 19, relativos a la posibilidad de realizar aprovechamientos cinegéticos al sur del Duero, a los tipos de zonificación, a los mecanismos de compensación de daños a la ganadería y al procedimiento de control de poblaciones. De ahí la conveniencia de aprobar un nuevo Plan, adaptado a las nuevas circunstancias sobre la situación de esta especie en la región. En efecto, si cuando se aprobó el Plan originario, Castilla y León contaba con ciento cuarenta y nueve manadas de lobo, prácticamente todas al norte del Duero, actualmente el número de manadas se ha visto incrementado en unas treinta; todas las provincias tienen núcleos reproductores y se ha producido una clara expansión hacia el sur y hacia la zona noroccidental, hasta el punto de que desde nuestra región los lobos han empezado 
a colonizar la región madrileña y de Castilla-La Mancha. Sí que es cierto que la densidad lobuna en la meseta cerealista se ha reducido, pero esta reducción se ha visto compensada con creces con el aumento en otras zonas.

Dos son, digamos, los principales problemas a los que se enfrenta la gestión de esta especie (canis lupus) en Castilla y León: la diversa consideración jurídica de la especie al norte y al sur del Duero, por una parte, y los conflictos con los ganaderos, por otra.

Respecto de la primera, la normativa básica estatal - de acuerdo con las Directivas europeas - incluye al lobo en diversas listas: por una parte, el Anexo II de la Ley 42/2007, de Patrimonio Natural y de la Biodiversidad, relativo a Especies animales y vegetales de interés comunitario para cuya conservación es necesario designar zonas especiales de conservación alude al canis lupus dejando claro que de las poblaciones españolas solamente se incluyen las del sur del Duero; y el Anexo V (Especies animales y vegetales de interés comunitario que requieren una protección estricta) incluye igualmente el canis lupus (excepto... las poblaciones españolas del norte del Duero); y por otra, el Anexo VI (Especies animales y vegetales de interés comunitario cuya recogida en la naturaleza y cuya explotación pueden ser objeto de medidas de gestión), incluye igualmente el canis lupus (poblaciones españolas al norte del Duero). Por lo tanto, por encima del Duero el lobo puede ser objeto de medidas de gestión, mientras que al sur debe ser estrictamente protegido. Una frontera tan débil como este río (con escaso caudal hasta su encuentro con el Pisuerga), muy fácilmente salvable, especialmente en verano, provoca una diferencia de régimen jurídico notable.

De acuerdo con ello, la gestión cinegética del lobo como técnica para mantener el equilibrio ecológico dinámico entre las poblaciones de este carnívoro y sus posibles presas de fauna silvestre sólo puede aplicarse en la zona 1, al norte del Duero (art. $6.1 \mathrm{a})$ ). Esta técnica se muestra como la más acertada para controlar esta población de la zona 1. Así lo señala el propio Plan: "El aprovechamiento cinegético ordenado y sostenible, en las comarcas donde la especie tenga la consideración de cinegética, se considera la mejor herramienta para la adecuada regulación poblacional de la especie" (art. 11.1). El aprovechamiento cinegético viene regulado en los arts. 15 y siguientes del Plan, y se concreta en la aprobación de cupos anuales de lobos cazables en función de la densidad en cada comarca. Estos cupos, con carácter general, no superarán el 10\% de los ejemplares (manadas) de la subzona o comarca, aunque se admite excepcionalmente la posibilidad de alcanzar el $18 \%$ en situaciones excepcionales de una especial siniestralidad 
en daños para la ganadería (art. 16). Lógicamente, los cupos pueden reducirse o incluso suspenderse en situaciones que afecten gravemente a la estabilidad poblacional de la especie.

Sin embargo, en la zona 2 no cabe la gestión cinegética; por eso el Plan se remite a las previsiones de la Ley de Biodiversidad: "Cuando resulte procedente realizar acciones de control poblacional en los territorios donde el lobo no tiene la consideración de especie cinegética, la dirección general competente en materia de medio natural podrá autorizar dichas acciones de control de conformidad con lo establecido en la Ley 42/2007, de 13 de diciembre, del Patrimonio Natural y de la Biodiversidad" (art. 12.1) y "de conformidad con lo dispuesto en el artículo 21 del Decreto 32/2015, de 30 de abril”, referido a los estrictos requisitos para la autorización de este tipo de acciones (art. 12.2).

Respecto a los conflictos con los ganaderos, tradicionales en esta tierra (basta recordar el famoso romance de la loba parda), uno de los objetivos del plan es "minimizar los efectos negativos que la especie pueda originar sobre el ganado, promoviendo la implantación de medidas de custodia y prevención de ataques". Para ello, el Plan dedica el capítulo I del Título IV (arts. 7-12) a las medidas de compatibilidad con la ganadería: aprobación futura de un Catálogo de buenas prácticas ganaderas en zonas loberas e incentivos para la adopción de medidas del catálogo, control de perros asilvestrados (incluso a través de medidas de lucha contra especies invasoras para evitar la hibridación) y, por supuesto, mediante medidas compensatorias en función del ganado afectado por los ataques de lobos, tal y como se desarrolla por la orden correspondiente, de 26 de mayo, sobre pagos compensatorios. Los controles poblacionales señalados mas arriba (cinegéticos —al norte del Duero - o no cinegéticos — al sur-) vendrán exigidos por un falta de equilibrio entre poblaciones loberas y presas potenciales silvestres, que facilita los ataques al ganado.

Además de estos dos ejes, el Plan de Gestión del lobo pretende actuar sobre las causas de mortalidad ilegal y accidental de la especie (arts. 13 y 14) disponiendo, p. ej., la adopción de medidas correctoras y compensatorias en las grandes infraestructuras que puedan afectar a estas poblaciones (pasos de lobos en autovías, etc.). Se posibilita, además, la captura en vivo de ejemplares para su posible traslocación a otros territorios en los que la especie haya estado representada tradicionalmente.

Finalmente, como gran novedad, el Plan pretende potenciar la figura del lobo como elemento del Patrimonio Natural de Castilla y León; se busca su aprovechamiento turístico (actualmente muy desarrollado para su avistamiento en zonas loberas, como la 
Sierra de la Culebra) y la generación de productos y servicios elaborados en estas zonas y derivados de ganado potencialmente expuestos a los ataques de estos cánidos.

Lógicamente, el éxito del Plan (el mantenimiento general de la especie, la colonización de nuevas áreas, especialmente al sur del Duero y al este de la región, la compatibilidad de la actividad ganadera con la buena salud de las manadas de lobos, etc.) parte de la base de un adecuado sistema informativo de seguimiento de las manadas, de un control estrecho del número de ejemplares, de una eficaz divulgación de las bondades de esta especie para el equilibrio de la ecología, etc.

Los objetivos y medios están claros. Sólo falta financiación suficiente y voluntad pública, pero también privada para alcanzar aquellos. Esperemos que los resultados sean los esperados por todos.

\subsection{El necesario complemento: la regulación de las ayudas compensatorias por ataques} de lobos

Como se ha señalado más arriba, el Plan del Lobo, tiene entro otros objetivos esenciales mantener la población de lobos de la región, haciéndola compatible con el aprovechamiento ganadero tradicional. Entre las medidas previstas por el Plan para ello figuran los pagos compensatorios por las cabezas de ganado afectadas por ataques de lobos (art. 10). En virtud de ello, pocos días después de la aprobación del Plan del Lobo se aprobó la Orden FYM/461/2016, de 26 de mayo, relativa a los pagos compensatorios derivados de los daños producidos por el lobo en las explotaciones ganaderas.

Se trata de una norma pormenorizada, que regula el régimen de los pagos compensatorios derivados de los daños ocasionados al ganado por el lobo en la Zona 2, esto es, al sur del Duero (art. 10.2 del Plan). No se alude a los daños ocasionados por lobos al norte del Duero porque, al tratarse de una especie cinegética, su reglamentación propia es la de caza, tal y como señala el art. 10.1 del Plan: "La responsabilidad por los daños causados por los lobos en la Zona 1, se determinará conforme a lo establecido en la norma en materia de caza".

La Orden promueve la instrucción de expedientes indemnizatorios en función de los ataques comprobados. Aunque solamente alude a los ataques de lobos, no de perros asilvestrados; hay que entender que no se puede exigir una diferenciación que los 
ganaderos no están en condiciones de valorar; constituye responsabilidad primigenia de la Administración impedir la existencia de estas manadas de perros, por lo que no se debe distinguir, a efectos de los pagos, entre una y otra especie. Por otra parte, la orden determina las cantidades a pagar en función del género (ovino, caprino, gallináceas, vacuno, equino, porcino), especie, edad y función (reproducción, producción de carne, de leche) de las cabezas de ganado muertas. Se señala, con claridad que el único daño compensable es la muerte (art. 1): ni las heridas, ni el estrés ni sus consecuencias son objeto de indemnización. Sin embargo, sí que se tiene en cuenta, no sólo el daño emergente, sino también el lucro cesante (art. 4).

En todo caso, para acceder a los pagos compensatorios, la orden exige el cumplimiento de un triple requisito: a) la previa inscripción en el Registro de Explotaciones Agrarias de Castilla y León, regulado mediante el Decreto 19/2015, de 5 de marzo, b) el cumplimiento de los programas de control, vigilancia y erradicación de enfermedades que puedan afectar a las especies ganaderas de acuerdo con la normativa aplicable al efecto y c) la identificación concreta de la explotación (art. 3.1). De esta forma, la Administración puede comprobar si el solicitante ha demostrado la diligencia necesaria en el cumplimiento de la normativa correspondiente. También se exige un mínimum de diligencia en la adopción de medidas de protección del ganado, porque los pagos no cubren los daños producidos en naves cerradas (o en montes de utilidad pública si no se han cumplido las obligaciones impuestas por la Ley de Montes (art. 3.2).

Se trata, por tanto, de un complemento indispensable para la correcta aplicación del Plan de Conservación y Gestión del Lobo en lo que se refiere a su compatibilidad con las explotaciones ganaderas tradicionales.

\section{Regulación de ayudas a la plantación de especies forestales de alto valor}

La Orden FYM/648/2016, de 6 de julio, establece las bases reguladoras para la concesión de ayudas al fomento de plantaciones de especies con producciones forestales de alto valor, cofinanciadas por el Fondo Europeo Agrícola de Desarrollo Rural (FEADER), en el marco del Programa de Desarrollo Rural de Castilla y León 2014-2020.

Como señala el Preámbulo, desde hace ya algunos años la Administración autonómica viene regulando la producción de materiales forestales a efectos de su comercialización: 
se trata de obtener productos de alta calidad en madera, hongos, piñón, resina o castañas para asegurar la viabilidad y éxito de las plantaciones correspondientes. De esta manera, se apunta a un modelo silvícola sostenible y a una mejora de la remuneración de los titulares de explotaciones forestales. Todo ello redunda en una mejora de la calidad de vida en el ámbito rural y de la oferta del mercado de productos silvícolas.

De acuerdo con ello, la Orden busca "fomentar la creación de plantaciones basadas en estos proyectos de innovación, con tres objetivos claramente diferenciados": producción de madera de calidad; altas producciones de piñón para consumo alimenticio (mediante pinos piñoneros injertados), y fomento de la truficultura (mediante plantaciones de determinadas especies micorrizadas previamente, destinadas a la recolección de hongos de alto valor comercial).

De esta forma, además de las finalidades económicas, las mencionadas ayudas tienen una clara finalidad ambiental (art. 2 B): la promoción del uso de recursos renovables en sustitución de materias primas que no cumplen con los criterios de sostenibilidad; la mejora del paisaje, introduciendo nuevos elementos en el mosaico agrícola; la mejora ecológica del territorio mediante el aumento de fijación del carbono atmosférico, de la protección de la fauna silvestre, de la calidad de los suelos, de la regulación del ciclo hídrico y de la mitigación del efecto invernadero que contribuye al cambio climático y conseguir repoblaciones potencialmente más estables que las tradicionales por utilizar especies mejor adaptadas al medio

Indudablemente, las ayudas cofinanciadas por la Unión Europea vendrán a potenciar la oferta de productos forestales de calidad y a poner en valor la riqueza silvícola de la región, manteniendo sus parámetros de calidad ambiental.

\section{Planes y estrategias no vinculantes}

\subsection{La II Estrategia de Educación Ambiental 2016-2020}

El Acuerdo 35/2016, de 9 de junio, de la Junta de Castilla y León, aprueba la II Estrategia de Educación Ambiental de Castilla y León 2016-2020.

La educación ambiental no constituye una competencia autónoma y distinta de las relativas a la regulación y aplicación en materia de medio ambiente. En efecto, toda 
actuación normativa en esta materia debe ir acompañada de los correspondientes procesos educativos, que permitan y faciliten su aplicación. No hay que olvidar que, en esta materia, la regulación suele ser innovadora y debe adaptarse constantemente a la evolución tecnológica, por lo que resulta imposible la aplicación correcta de la normativa ambiental sin un aprendizaje suficiente.

Por otra parte, la educación ambiental constituye un elemento esencial para lograr que la protección del medio ambiente no sea un objetivo de la exclusiva responsabilidad de los poderes públicos. A través de una educación ambiental correcta se puede y se debe corresponsabilizar a la población del papel que cada miembro del entramado social tiene en la salvaguarda del medio ambiente.

En este sentido, conviene que la Administración autonómica haga llegar, al sector educativo público y privado y a las Administraciones con competencias ambientales la necesidad de informar y formar en esta materia: ayuntamientos, diputaciones, universidades, centros docentes públicos y privados y educadores en general.

Además, desde un punto de vista estrictamente presupuestario, la sensibilización de la población con los valores ambientales hace de los ciudadanos, por corresponsables, colaboradores en la salvaguarda del interés general ambiental, de forma que la Administración puede eludir costes gracias a dicha sensibilización ciudadana. Esta ventaja, en una situación de crisis económica y de políticas presupuestarias restrictivas, constituye un elemento que deben tener en cuenta los poderes públicos. En efecto, como explica la propia Exposición de Motivos del Plan, los recientes cambios económicos han implicado cambios significativos en la forma de concebir y desarrollar la educación ambiental: nuevas necesidades, la conveniencia de intensificar la vinculación con la gestión ambiental, incrementar la eficacia, mejorar la colaboración entre agentes y diversificar la financiación.

Con este cambio del supuesto de hecho, el Acuerdo 35/2016, de 9 de junio, por el que se aprueba la II Estrategia de Educación Ambiental, viene a sustituir a la I Estrategia de Educación Ambiental 2003-2007, aprobada mediante Acuerdo 3/2003, de 2 de enero, de la Junta de Castilla y León.

El contenido innovador de esta nueva Estrategia, justificada en los antecedentes señalados, se centra en las nuevas "Orientaciones Estratégicas", que son, en síntesis, las siguientes: "Mejorar la colaboración con instituciones y entidades, desarrollando 
proyectos comunes, fomentando sinergias y optimizando los recursos disponibles. Diversificar las fuentes de financiación de la educación ambiental. Incrementar la colaboración público-privada. Favorecer la viabilidad económica de las empresas de educación ambiental. Reforzar la educación ambiental en los centros escolares y universidades. Dedicar una atención especial al medio rural de Castilla y León. Generalizar la implantación de buenas prácticas ambientales en las Administraciones Públicas, empresas privadas, centros educativos y universidades. Impulsar la información, comunicación y participación en todas las acciones y decisiones de gestión ambiental y garantizar la accesibilidad y comprensión de la información ambiental".

Aunque se trata de un programa ciertamente ambicioso, estamos, lógicamente, ante una disposición no vinculante, que carece de naturaleza normativa, pero que puede cumplir una importante función indicativa, directiva de las futuras actuaciones de las Administraciones públicas en la materia, tanto en lo que se refiere a la prestación directa de servicios educativos, inclusivos de la información ambiental imprescindible, como en lo relativo a las ayudas y subvenciones dirigidas a la realización de actividades de educación ambiental por los distintos operadores educativos.

\subsection{La Estrategia de Producción Ecológica 2016-2020}

El Acuerdo 22/2016, de 5 de mayo, de la Junta de Castilla y León, aprueba el Plan Estratégico de Producción Ecológica de Castilla y León 2016-2020.

El Plan Estratégico de Producción Ecológica de Castilla y León viene a responder a las necesidades de potenciar los productos alimenticios de la región cuya producción se haya llevado a cabo mediante el cumplimiento de estándares de elevada calidad ambiental.

Este plan se dicta en ejercicio de las competencias exclusivas de la Comunidad Autónoma en agricultura y en denominaciones de origen y otras protecciones de calidad (art. $71.1 .14^{\circ}$ y $15^{\circ}$ del Estatuto de Autonomía) y conecta directamente con las previsiones de la Ley 1/2014, de 19 de marzo, agraria de Castilla y León, sobre la agricultura ecológica (arts. 133-135). Finalmente, no puede olvidarse la legitimación europea de esta regulación. La producción ecológica constituye el objeto de la regulación europea contenida en el Reglamento (CE) $n^{\circ}$. 834/2007, del Consejo, de 28 de junio, sobre producción, distribución, control y etiquetado de productos ecológicos. 
El Plan de Producción Ecológica viene a responder a una creciente demanda social de productos ecológicos: la preocupación por el medio ambiente y la salud son cada vez mayores y la producción ecológica permite asegurar la calidad del alimento y es garantía de salud para el consumidor. No hay que olvidar que los sellos de calidad vinculados a la producción e industria agropecuaria ecológica constituyen un incentivo para que los productores asuman formas de producción acordes al interés público; el sello de producción ecológica constituye, por tanto, una manifestación de la actividad administrativa de fomento o estímulo, sin necesidad de desembolso económico para la Administración autora de la regulación: los productores se ven incentivados, no por la remuneración o subvención pública, sino por el acceso a un sector del mercado que está dispuesto a pagar más a cambio de productos de calidad contrastada.

La regulación del Plan de Producción Ecológica busca, por tanto, alcanzar una serie de objetivos de interés público: la obtención, por parte de los productores, de ayudas comunitarias para la conversión a la agricultura ecológica o para su mantenimiento (que constituyen un estímulo añadido para la participación de los agricultores en este sistema de producción). Además, las posibilidades de expansión de estas técnicas "limpias" pueden facilitar la consecución de algunos objetivos del sector agrario en esta región, como son la incorporación de jóvenes a este sector y el consecuente relevo generacional mediante la generación de empleo, la competitividad y sostenibilidad ambiental de las explotaciones, la diversificación de las actividades, y la generación de riqueza en el medio rural.

El Plan parte de un dato incontestable: por una parte (y sorprendentemente), la producción ecológica en Castilla y León es menor que en el resto de España. Por otra parte - y esto es una ventaja incontestable - en nuestra región la producción extensiva (agrícola y ganadera) es mayoritaria, lo que requiere menores cambios en el sistema productivo para su transformación en un sistema ecológico, y por tener menor uso de insumos, los agricultores no dependen tanto de la variación de precios. Además, existe un importante número de pequeñas explotaciones agrícolas que tienen asociada su actividad a sistemas de elaboración de productos tradicionales, cercanos a los ecológicos. En definitiva, es relativamente fácil y asequible plantear la conversión de la agricultura y ganadería tradicionales en "ecológica", esto es, en actividades susceptibles de ser oficialmente cualificadas. 
Desde este punto de vista, el Plan incluye numerosas medidas, distribuidas en cuatro grandes áreas de actuación: la producción, la transformación, el mercado y, finalmente, el equilibrio del sector ecológico: esta última exige adoptar medidas de transparencia, formación, promoción e información, investigación y seguimiento y control.

\subsection{La Estrategia de Regeneración Urbana}

El Acuerdo 40/2016, de 23 de junio, de la Junta de Castilla y León, aprueba la Estrategia de Regeneración Urbana en Castilla y León.

Aunque la Estrategia tiene su marco jurídico en la regulación urbanística (Ley 7/2014, de 12 de septiembre, de medidas sobre rehabilitación, regeneración y renovación urbana, y sobre sostenibilidad, coordinación y simplificación en materia de urbanismo, y el Decreto 6/2016, de 3 de marzo por el que se modifica el Reglamento de Urbanismo de Castilla y León), es innegable que esta regeneración urbana tiene una estrecha vinculación con el medio ambiente. Lo pone de manifiesto la propia Exposición de Motivos de la Estrategia: "en el contexto actual, la regeneración urbana debe entenderse como la recuperación física y funcional de las zonas obsoletas de pueblos y ciudades mediante el establecimiento de condiciones ambientales, sociales y económicas que promuevan un desarrollo sostenible y una mejora en la calidad de vida de sus habitantes, y constituye en la actualidad el objetivo prioritario de la acción pública urbanística del Gobierno regional".

El desarrollo urbano sostenible ha pasado a ser, por tanto, un objetivo de primer orden en el desarrollo urbanístico regional (y estatal). Y este desarrollo sostenible va unido, como no podía ser menos, a la calidad de vida de los ciudadanos de la región: no se trata de incrementar la oferta de vivienda en Castilla y León (claramente sobredimensionada por el urbanismo "constructor" de los primeros años del siglo) sino de recuperar zonas, de hacer más habitables las ciudades y pueblos, el marco cívico, evitando el crecimiento salvaje o el anquilosamiento urbanístico. 